\title{
Coherent photons with angular momentum in a helical afterburner
}

\author{
Erik Hemsing (e) \\ SLAC National Accelerator Laboratory, Menlo Park, California 94025, USA
}

(Received 15 November 2019; accepted 28 January 2020; published 11 February 2020)

\begin{abstract}
We analytically examine several important characteristics of coherent radiation emitted from a relativistic electron beam in a helical undulator. The goal is to highlight specific attributes relevant to the production of bright $\mathrm{x}$-rays with angular momentum in a free-electron laser (FEL) afterburner. The farfield complex electric field distribution is derived, and for small forward emission angles, it is confirmed that the spin and orbital components of angular momentum in the radiation are separable, additive, and aligned with the handedness of the electron motion. The coherent energy radiated at harmonics $h$ from a bunched electron beam is then calculated, and used to evaluate the emission energy from a FEL afterburner configuration. Results show that an afterburner undulator with $N_{u} \gg 1$ periods and of sufficient strength will produce harmonic pulses with energy $\sim 1 / N_{u}^{h-1}$ times that of the fundamental, depending on the Fresnel number $N=k \sigma_{x}^{2} / L_{u}$ of the radiating electron beam.
\end{abstract}

DOI: 10.1103/PhysRevAccelBeams.23.020703

\section{INTRODUCTION}

In addition to spin angular momentum (SAM), electromagnetic waves can also carry a projection of orbital angular momentum (OAM) along the axis of propagation. This can be realized, for example, in the spherical waves emitted from multipoles [1], though in such cases a distinction between the spin and orbital parts is not clear. More recently, Allen et al., [2] showed that for Laguerre Gaussian (LG) modes, which are cylindrical solutions to the paraxial wave equation, the spin and orbital components are separable and that the aziumuthal modes carry $l \hbar$ units of OAM per photon, where $l$ is an integer. The OAM is attributed to the helical phase fronts and corresponding azimuthal momentum about an on-axis phase singularity. The scientific impact of such beams has been a topic of intense research (see, e.g., [3-8]), including numerous studies on the precise manner in which the OAM may manifest in interactions with physical systems [9-15].

Motivated by the myriad of physical and practical applications, there has been growing interest in the production of bright OAM light at sub-optical wavelengths in free electron lasers (FELs). FELs use relativistic electron beams propagating through magnetic undulators to produce intense pulses of light down to Angstrom wavelengths and at femtosecond pulse durations [16-18]. Sasaki and McNulty [19] first pointed out that the harmonic radiation

Published by the American Physical Society under the terms of the Creative Commons Attribution 4.0 International license. Further distribution of this work must maintain attribution to the author(s) and the published article's title, journal citation, and DOI. in helical undulators (in which the electrons follow a helical path) carried a helical phase, and thus that helical undulators might serve as intense sources of OAM light. While it has long been known that the harmonic radiation is off-axis (i.e., Refs. [20-22]), the predicted helical phase was first experimentally measured only recently in spontaneous undulator emission by Bahrdt and colleagues [23], and later in coherent emission from a density modulated (bunched) electron beam [24]. Subsequent demonstration experiments confirmed the basic principles $[25,26]$.

Here, to provide theoretical foundation for emerging science and applications with coherent OAM light produced in modern FELs, we examine several critical properties of the radiation from helical undulators, including the polarization, phase handedness, and total pulse energy of coherent emission. To provide a cohesive framework, we first provide a general expression for the far-field angular emission spectrum from a single electron. At small forward emission angles and within certain limits, we confirm previous conclusions that the SAM and OAM components add, and are in the same direction as the electron motion $[27,28]$. An important finding is that this conclusion is reliable only when the undulator has sufficiently many periods $\left(N_{u} \gg 1\right)$ and is sufficiently strong in undulator field strength. We then use these results to derive simple expressions for the energy of coherent OAM radiation from a bunched electron beam (e-beam) for the first few harmonics. We then examine the emission energy in an FEL OAM afterburner arrangement as proposed in Ref. [24], in which a helical undulator is placed downstream of an FEL to produce coherent OAM pulses from the spent, bunched e-beam. We then look at an example case that produces $20 \mathrm{MW}$ x-ray OAM beams that, if 
focused to a $100 \mathrm{~nm}$ spot, can reach intensities of $10^{15-16} \mathrm{~W} / \mathrm{cm}^{2}$ (electric fields $10^{9} \mathrm{~V} / \mathrm{cm}$, or 1 a.u.) and may be of use to examine, for example, dipole-forbidden electronic transitions in localized samples near the welldefined phase singularity [29,30].

\section{RADIATED FIELD DISTRIBUTION}

Consider a helical magnetic undulator field described by the vector

$$
\vec{B}=B_{0}\left[\cos \left(\omega_{u} t\right) \hat{x} \mp \sin \left(\omega_{u} t\right) \hat{y}\right]
$$

where $\omega_{u}=k_{u} v_{z}$ is the angular frequency of rotation of a particle with constant $z$-velocity $v_{z}$, and $z=v_{z} t=\beta_{z} c t$. The upper sign refers to a left-handed (LHU) undulator, and the lower sign refers to a right-handed (RHU) undulator, corresponding to the evolution of the $\vec{B}$ field vector along $z$. The coordinate vector that describes the evolution of an electron is

$$
\vec{w}(t)=\frac{K}{\gamma k_{u}}\left[ \pm \sin \left(\omega_{u} t\right) \hat{x}+\cos \left(\omega_{u} t\right) \hat{y}\right]+\beta_{z} c t \hat{z} .
$$

The undulator field strength (in cgs units) is $K=$ $q B_{0} / k_{u} m c^{2}$. The electron trajectory has the same handedness as the undulator field. The scaled velocity vector of the electron is then

$\vec{\beta}(t)=\frac{d \vec{w}(t)}{c d t}=\frac{K}{\gamma}\left[ \pm \cos \left(\omega_{u} t\right) \hat{x}-\sin \left(\omega_{u} t\right) \hat{y}\right]+\beta_{z} \hat{z}$.

These vectors are used to calculate the properties of the radiation from electron spiraling through the undulator.

We first define the Fourier transform of the temporal electric field,

$$
\vec{E}(\omega)=\frac{1}{\sqrt{2 \pi}} \int \vec{E}(t) e^{i \omega t} d t
$$

Far from the electron, the electric field radiated from within the undulator with $N_{u}$ periods is given in the frequency domain by (e.g., Ref. [31])

$\vec{E}(\omega)=\frac{-i q \omega e^{i \omega r / c}}{\sqrt{2 \pi} c r} \int_{-\frac{N_{u} \pi}{\omega_{u}}}^{\frac{N_{u} \pi}{\omega_{u}}}[\hat{n} \times(\hat{n} \times \vec{\beta})] e^{i \omega(t-\hat{n} \cdot \vec{w} / c)} d t$,

where

$$
\hat{n}=\vec{r} / r=\sin \theta \cos \phi \hat{x}+\sin \theta \sin \phi \hat{y}+\cos \theta \hat{z}
$$

is the unit vector pointing from the origin to the observation point, $\theta$ is the forward inclination angle from the $z$-axis, and $\phi$ is the azimuthal angle.
Combining Eqs. (3) and (6), the combination of cross products within the integral gives the different components of the field polarization,

$$
\begin{aligned}
\hat{n} \times(\hat{n} \times \vec{\beta}) \\
=\left(\begin{array}{c}
\mp \alpha_{1} \cos \left(\omega_{u} t\right)-\alpha_{2} \sin ^{2} \theta \sin \left(\omega_{u} t\right)+\beta_{z} \alpha_{3} \cos \phi \\
\alpha_{1} \sin \left(\omega_{u} t\right) \pm \alpha_{2} \sin ^{2} \theta \cos \left(\omega_{u} t\right)+\beta_{z} \alpha_{3} \sin \phi \\
\frac{K}{\gamma} \alpha_{3}\left[ \pm \cos \phi \cos \left(\omega_{u} t\right)-\sin \phi \sin \left(\omega_{u} t\right)\right]-\beta_{z} \sin ^{2} \theta
\end{array}\right)
\end{aligned}
$$

with

$$
\begin{aligned}
& \alpha_{1}=\frac{K}{\gamma}\left(\cos ^{2} \theta+\sin ^{2} \theta \cos ^{2} \phi\right), \\
& \alpha_{2}=\frac{K}{\gamma} \cos \phi \sin \phi, \quad \alpha_{3}=\cos \theta \sin \theta .
\end{aligned}
$$

The terms within the exponential are

$\frac{\omega}{c} \hat{n} \cdot \vec{w}=\xi\left[\sin \phi \cos \left(\omega_{u} t\right) \pm \cos \phi \sin \left(\omega_{u} t\right)\right]+\omega t \beta_{z} \cos \theta$

where we define the dimensionless parameter

$$
\xi=\frac{\omega K}{\gamma k_{u} c} \sin \theta .
$$

Using series expansions, we can write the $e^{i \omega(t-\hat{n} \cdot \vec{w} / c)}$ as a sum over Bessel functions and compute a number of straightforward integrals (see the Appendix in Sec. A). Assuming an undulator with many periods, $N_{u} \gg 1$, the spectrum is peaked at the harmonic frequencies

$$
\omega_{h}(\theta)=\frac{h \omega_{u}}{1-\beta_{z} \cos \theta}
$$

where $h$ is the harmonic. Near the harmonics, solutions for each of the field components are

$$
\vec{E}(\omega)=E_{0}(\omega)\left(\begin{array}{c}
\mp \alpha_{1} \zeta_{1}-\alpha_{2} \zeta_{2} \sin ^{2} \theta+\beta_{z} \alpha_{3} \zeta_{3} \cos \phi \\
\alpha_{1} \zeta_{2} \pm \alpha_{2} \zeta_{1} \sin ^{2} \theta+\beta_{z} \alpha_{3} \zeta_{3} \sin \phi \\
\frac{K}{\gamma} \alpha_{3}\left[ \pm \zeta_{1} \cos \phi-\zeta_{2} \sin \phi\right]-\beta_{z} \zeta_{3} \sin ^{2} \theta
\end{array}\right)
$$

where the term common to all the complex field vectors is

$$
E_{0}(\omega)=\frac{-i q \omega}{\sqrt{2 \pi} c r} \frac{N_{u} \pi}{\omega_{u}} e^{i \omega r / c} \operatorname{sinc}\left[N_{u} \pi h\left(\frac{\omega}{\omega_{h}(\theta)}-1\right)\right],
$$

and 


$$
\begin{aligned}
& \zeta_{1}=( \pm 1)^{h+1}\left[e^{\mp i(h-1) \phi} J_{h-1}(\xi)+e^{\mp i(h+1) \phi} J_{h+1}(\xi)\right] \\
& \zeta_{2}=i( \pm 1)^{h+1}\left[e^{\mp i(h-1) \phi} J_{h-1}(\xi)-e^{\mp i(h+1) \phi} J_{h+1}(\xi)\right] \\
& \zeta_{3}=2( \pm 1)^{h} e^{\mp i h \phi} J_{h}(\xi) .
\end{aligned}
$$

In general, the fields have rich spatial-frequency correlations that lead to a mixing of the spin and orbital angular momentum components. In most cases, however, many of the less-significant terms in (12) and (14) can be ignored when evaluating the dominant forward emission near the axis.

\section{SMALL ANGLE SOLUTIONS}

At small forward angles $\theta \ll 1$ and for the first few harmonics, the polarization and transverse distributions become well-separated. Namely, if the Bessel argument is small, $\xi=\frac{2 h K \gamma \theta}{1+K^{2}}=2 h K \gamma_{z}^{2} \theta / \gamma<1$ then only the lowest order Bessel functions in (14) contribute,

$\zeta_{1} \approx( \pm 1)^{h+1} e^{\mp i(h-1) \phi} J_{h-1}(\xi), \quad \zeta_{2} \approx i \zeta_{1}, \quad \zeta_{3} \approx 0$.

For $\theta \ll 1$ from (8) we can write $\alpha_{1} \approx \frac{K}{\gamma}$ and $\alpha_{3} \approx \theta$. The individual field components then have much simpler forms, and the total field vector in (12) is expressed compactly as

$$
\vec{E}(\omega) \approx \mp E_{0}(\omega) \frac{K}{\gamma} \zeta_{1}\left(\begin{array}{c}
1 \\
\mp i \\
-\theta e^{\mp i \phi}
\end{array}\right) .
$$

\section{ANGULAR MOMENTUM}

From Eq. (16), the transverse components of the radiated field are $\pm \pi / 2$ out of phase, $E_{y}(\omega)=\mp i E_{x}(\omega)$, describing circularly polarized waves. For harmonics $h>1$, they also have a pure azimuthal dependence $e^{\mp i(h-1) \phi}$ from $\zeta_{1}$. Combined with Eq. (13), the transverse fields can therefore be summarized as

$$
\vec{E}_{\perp} \rightarrow\left(\begin{array}{c}
1 \\
i \sigma
\end{array}\right) e^{i(k z-\omega t+l \phi)}
$$

where $k=\omega / c$, and the spin and orbital contributions are

$$
\sigma=\mp 1, \quad l=\mp(h-1) .
$$

This separation of spin and orbital components in the small angle approximation bears a strong similarity with the behavior predicted in previous studies of paraxial and nonparaxial beams [32]. Here, inspection shows that at a fixed point in space, the polarization rotates in time in the same direction as the electron motion [33]. The $z$-component of the spin is then $S_{z}=\sigma \hbar$.
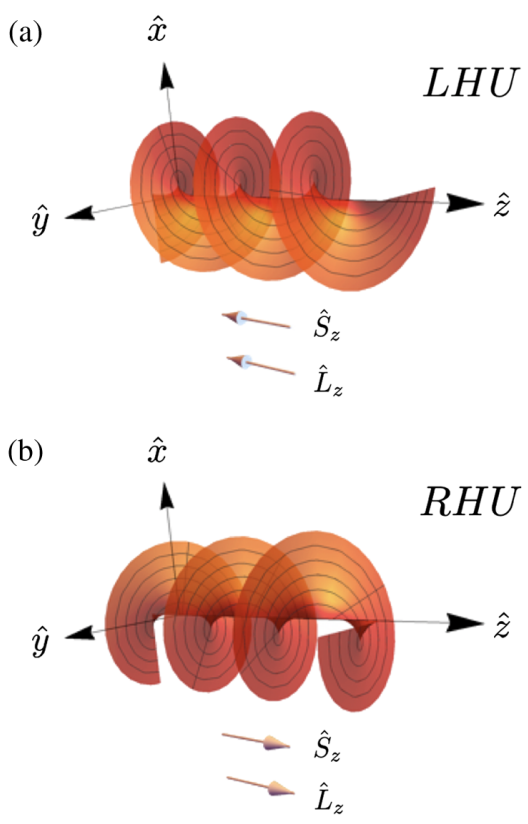

FIG. 1. Constant phase contours for second harmonic emission from (a) left-handed undulator (LHU) and (b) right-handed undulator (RHU). Also indicated are the corresponding projections of spin $\hat{S}_{z}$ and angular $\hat{L}_{z}$ momentum for the electromagnetic fields, which point in the same direction as the classical orbital angular momentum of the electrons on their helical trajectory through the undulator.

The spatiotemporal correlation with $\phi$ describes a phase that winds helically about the axis of propagation for harmonics $h>1$. If $l<0$, (i.e., an LHU) the surface of constant phase in $(z, \phi)$ corkscrews in a right-handed sense for a fixed time. This is shown in Fig. 1(a). However, the Poynting vector, which is normal to the phase front and describes the direction of momentum flow, winds in a lefthanded sense along $z$. Thus, in agreement with the convention of previous studies [2,3], this describes a field which has a projection of OAM in the $-z$ direction. This is consistent if we recall that the $z$-component of the quantum mechanical operator for orbital angular momentum is represented in the position space as $\hat{L}_{z} \rightarrow \frac{\hbar}{i} \frac{\partial}{\partial \phi}$. The OAM of the harmonic fields is thus $L_{z}=l \hbar$, and has the same sign as the SAM. It is concluded that the two contributions to the total angular momentum add in the described limits, and are related by the harmonic frequency [28],

$$
J_{z}=S_{z}+L_{z}=\mp h \hbar
$$

Note that one cannot readily flip the sign of the polarization without also changing the direction of OAM for helical undulator emission.

We stress that the clean separation of angular momentum components in Eq. (18) comes from the small-angle solutions in Eq. (16), but that isolation of singular 

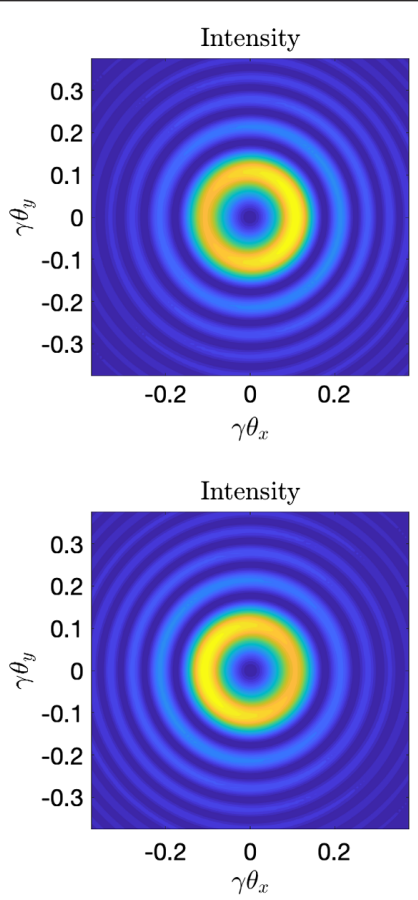
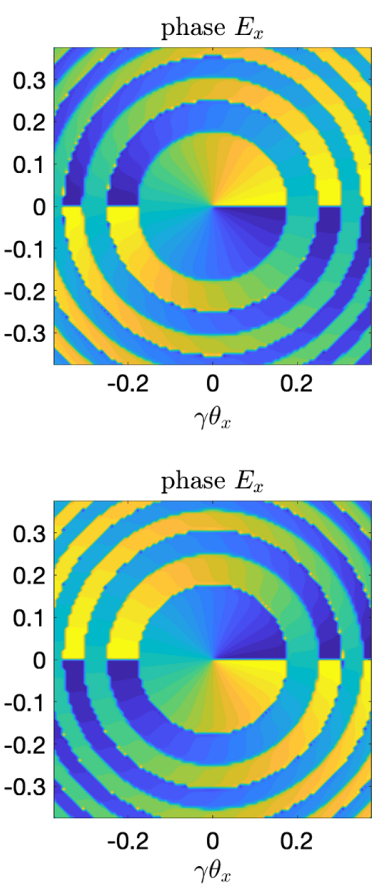
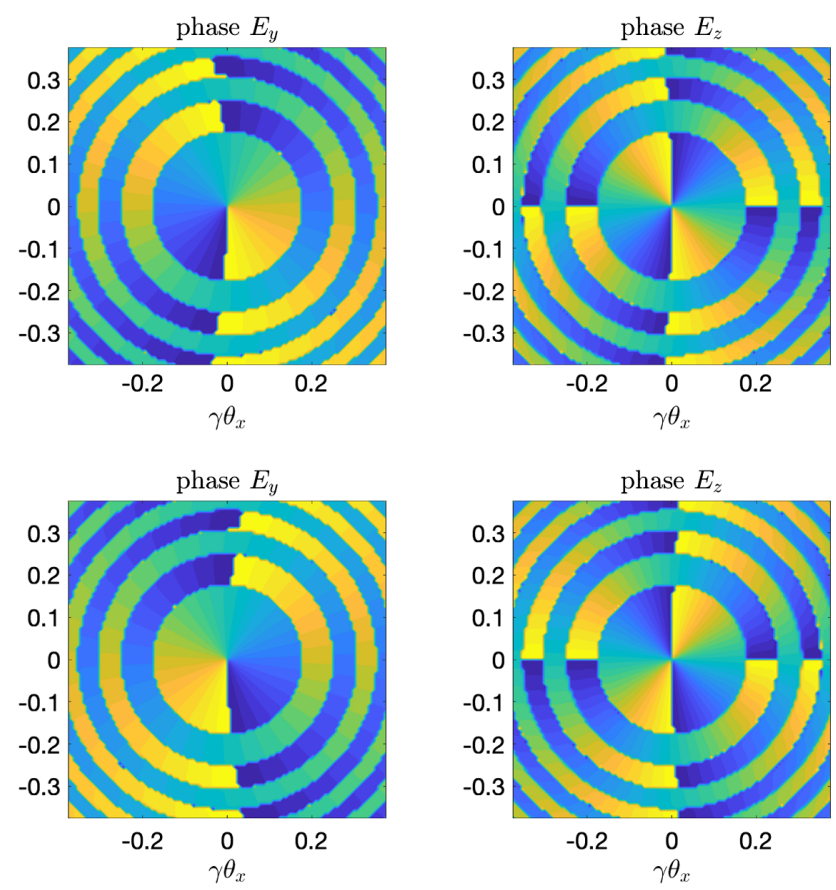

FIG. 2. Top: Second harmonic emission calculated from the full field expression in Eq. (5) with $N_{u}=100, K=2.3, \gamma=7828$ $(4 \mathrm{GeV}), \lambda_{u}=3.9 \mathrm{~cm}, \lambda=1 \mathrm{~nm}$. Parameters are inspired by LCLS-II project at SLAC [36]. Top: LHU produces $\sigma=-1$ polarization and an $l=-1$ vortex. Bottom: An RHU produces $\sigma=+1$ polarization and an $l=+1$ vortex.

contributions is not as simple in general. In fact, even for the cases where the small angle approximation is valid, more than one OAM mode may be present, particularly for high harmonics. For the second harmonic at resonance, one can see by inspection of Fig. 2, for example, that there is a slight azimuthal asymmetry in the intensity profiles that indicates the presence of more than one azimuthal mode. This is ultimately the result of the complicated angular spectral coupling in the full field expressions. Decomposing the field profiles into the constituent modes (e.g., as in [35]), the profile only approaches a single azimuthal mode for sufficiently large $N_{u}$ and $K$, as shown in Fig. 3.

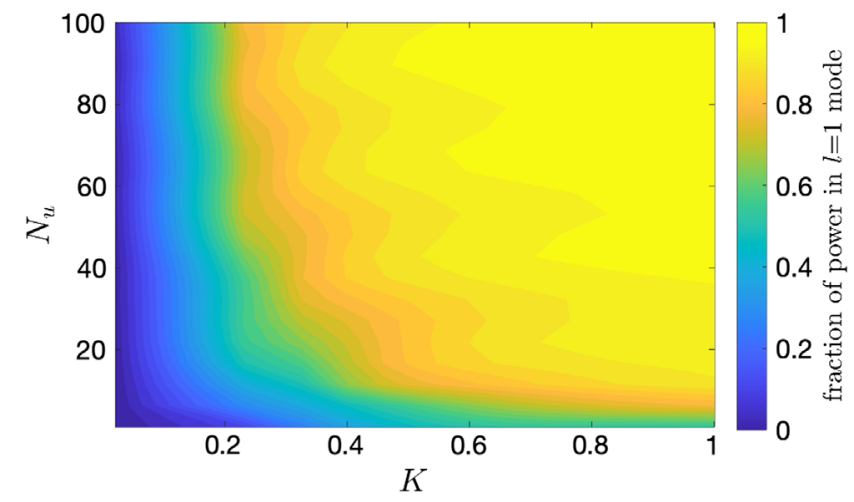

FIG. 3. Fraction of power in the $l=1$ mode (integrated over radial modes) emitted at the second harmonic in a RHU for a single electron with $\gamma=7828, \lambda=1 \mathrm{~nm}$. It reaches $98 \%$ at $N_{u}=100$, and $K=1$.
Finally, we note that the annular shape of the harmonic radiation profile is due, in part, to the Bessel function dependence on $\theta$, so the emitted fields from a single electron are not strictly LG modes, even at a single frequency. However, for sufficiently large $N_{u}$ and $K$, it is straightforward to show that the coherent emission from a bunched beam of electrons with a round Gaussian transverse profile can produce an exact LG mode. Consider the $L G_{p, l}=L G_{0, \mp 1}$ mode at the second harmonic. As examined in the next section, if the transverse e-beam size satisfies $\sigma_{x}>\gamma_{z} \sqrt{N_{u}} / k$, then the narrow forward emission angles are dominated by the e-beam and not the undulator emission kernel. This further simplifies the complicated angular-spectral coupling in the full field expression such that the transverse field distribution can be written as

$$
E_{\perp} \propto \theta e^{-\frac{\left(\sigma_{x} k \theta\right)^{2}}{2}} e^{i k z \mp i \phi}=\frac{1}{\sigma_{x} k}\left(\frac{\sqrt{2} \rho}{w_{0}}\right) e^{-\frac{\rho^{2}}{w_{0}^{2}}} e^{i k z \mp i \phi} .
$$

Spherical coordinates have been converted to cylindrical coordinates with $\rho=z \tan \theta \approx z \theta$. In this form, the field is precisely that of an $L G_{0, \mp 1}$ mode with waist size $w_{0}=$ $\sqrt{2} z / \sigma_{x} k$. Therefore, for coherent radiation in the proper limits, it is suitable to describe the OAM content at the lower harmonics in terms of the $L G$ mode basis.

\section{COHERENT RADIATION}

The radiated spectral energy distribution is given in general by [31] 


$$
\frac{d W}{d \Omega d \omega}=\frac{c}{2 \pi}|r \vec{E}(\omega)|^{2} .
$$

Using this equation and the field components in (12), we can calculate an explicit, general expression for the energy radiated per unit solid angle, per unit frequency in an undulator. Near a harmonic $h$, the answer has already been given by Colson [20]. At small forward angles and near the first couple of harmonics, the approximate field expression in Eq. (16) produces

$$
\begin{aligned}
\frac{d^{2} W_{h}}{d \Omega d \omega} \approx & \frac{q^{2} N_{u}^{2}}{2 c} \operatorname{sinc}\left[\pi N_{u} h\left(\frac{\omega}{\omega_{h}(0)}\left(1+\theta^{2} \gamma_{z}^{2}\right)-1\right)\right]^{2} \\
& \times\left(\frac{2 h K \gamma}{1+K^{2}}\right)^{2} J_{h-1}^{2}\left(\frac{2 h K \gamma \theta}{1+K^{2}}\right) .
\end{aligned}
$$

For a long beam of $N_{e}$ particles, the coherent emission is calculated in general with

$$
\frac{d^{2} W_{C}}{d \Omega d \omega}=N_{e}^{2} F(\omega) \frac{d^{2} W_{h}}{d \Omega d \omega}
$$

where $F(\omega)$ is the e-beam form factor, calculated from the Fourier transform of the spatial charge distribution,

$$
F(\omega)=\left|\int f(\vec{x}) e^{-i \omega \hat{n} \cdot \vec{x} / c} d \vec{x}\right|^{2} .
$$

Let us assume a transversely round Gaussian beam with rms radius $\sigma_{x}$ and a flattop longitudinal current profile of length $\tau$,

$$
f(\vec{x})=\frac{1}{2 \pi \sigma_{x}^{2}} e^{-\left(x^{2}+y^{2}\right) / 2 \sigma_{x}^{2}} \frac{\Pi(t / \tau)}{\tau}\left[1+2 b \cos \omega_{b} t\right]
$$

where $b \ll 1$ is the bunching factor at the frequency $\omega_{b} \gg 1 / \tau$, and $\Pi(t / \tau)$ is the rectangle function that is unity for $|t| \leq \tau / 2$ and zero otherwise. The form factor near the bunching frequency is [37]

$$
F(\omega)=b^{2} \sin ^{2}\left[\frac{\tau}{2}\left(\omega_{b}-\omega \cos \theta\right)\right] \exp \left[-\sigma_{x}^{2} \frac{\omega^{2}}{c^{2}} \sin ^{2} \theta\right] .
$$

The sinc term captures the impact of the e-beam length on the bunching spectrum, and the Gaussian term describes the forward angular projection effects due to the finite transverse e-beam size. For small forward angles $\theta \ll 1$, the form factor of the e-beam restricts the forward emission to angles that satisfy

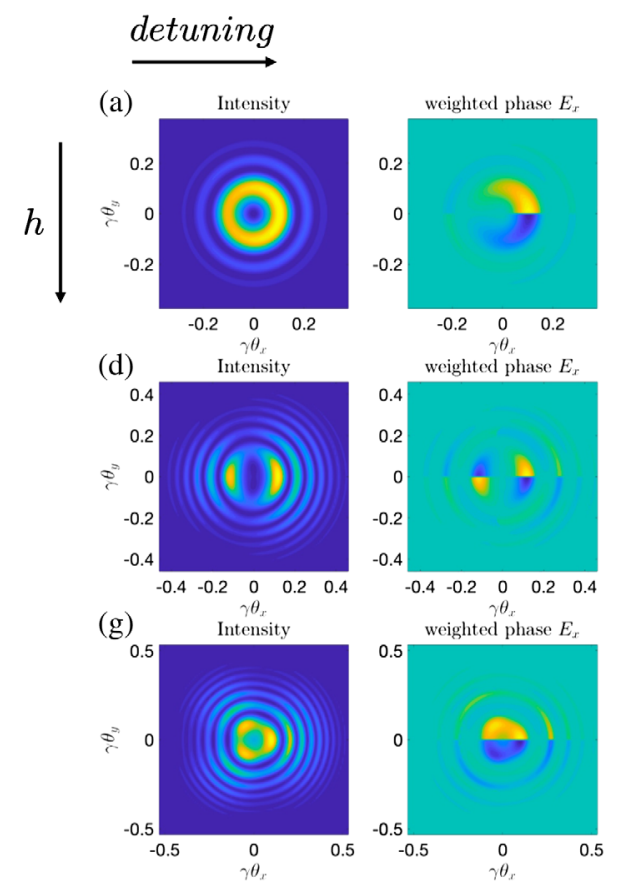

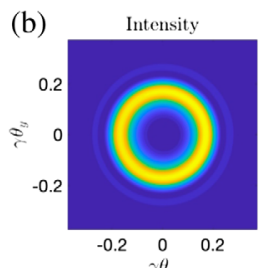
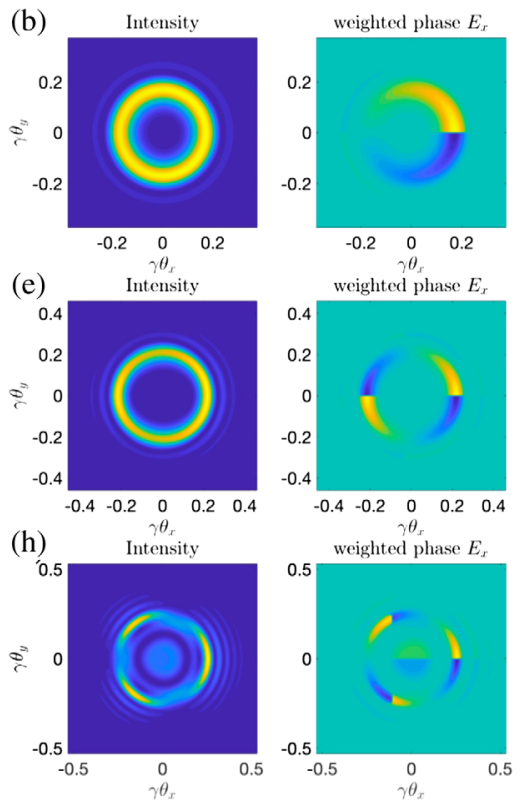
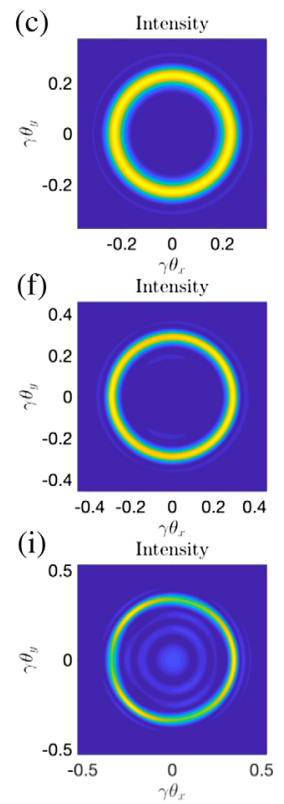
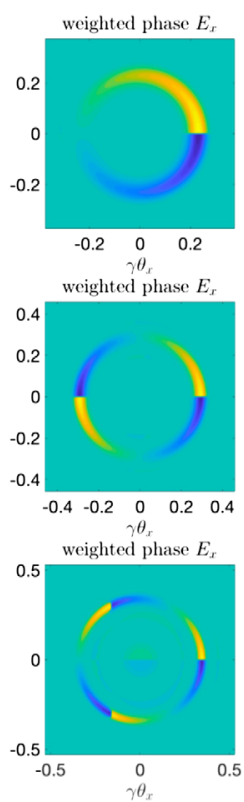

FIG. 4. Coherent OAM emission for different LHU harmonics (rows) and undulator detuning (columns). Top to bottom the rows are harmonics $h=2, h=3$, and $h=4$, respectively, with resonant undulator strengths $K_{2}=2.3, K_{3}=2.9$, and $K_{4}=3.4$. The $4 \mathrm{GeV}$ beam is bunched at $\lambda=1 \mathrm{~nm}$, and the undulator has $N_{u}=100$ periods of $\lambda_{u}=3.9 \mathrm{~cm}$. The detuning increases left to right according to $K \rightarrow K_{h}(1-\delta)$, with $\delta=0,1 / 4 N_{u}$, and $1 / 2 N_{u}$. The annular intensity profile is observed to expand and become more symmetric with detuning, which, with the phase, indicates that the power is directed into a single OAM mode. The phase is weighted by the intensity for better visibility. A small $\sigma_{x}=5 \mu \mathrm{m}$ e-beam $(N=0.04)$ is modeled to highlight the effect of detuning without strongly suppressing the output. 


$$
\theta<c / \sigma_{x} \omega
$$

For comparison, the intrinsic forward angular spread of the single particle undulator radiation is $\Delta \theta \approx 1 / \gamma_{z} \sqrt{N_{u}}$ near resonance.

Figure 4 shows the coherent intensity and phase profiles from full solutions for different harmonics and at different undulator detunings; i.e., at undulator strengths $K$ such that the on-axis undulator resonant frequency $\omega_{h}(0)=$ $2 h \gamma^{2} \omega_{u} /\left(1+K^{2}\right)$ in Eq. (11) is higher than the microbunching frequency, $\omega_{h}(0)>\omega_{b}$. Detuning the undulator resonance by slightly reducing $K$ pushes the radiation offaxis. For the higher harmonics, this has the beneficial effect of making the transverse profiles more rotationally symmetric, thus increasing the power in a single azimuthal mode. Detuning off-axis may also increase the emitted field energy as long as the annular intensity peak is at an angle that satisfies Eq. (27). The total emitted energy dependence on detuning is shown in Fig. 5 for parameters of the LCLS-II project at SLAC [36], where results indicate that approximately $1 \mathrm{MW}$ of peak power is emitted into the $l= \pm 1$ mode with the baseline design. If the electron beam size can be reduced to $\sigma_{x}=5 \mu \mathrm{m}(N=0.04)$, this increases to $20 \mathrm{MW}$.

Integrating Eq. (23) over frequency and angle gives the total radiated energy

$$
W_{C}=N_{e}^{2} \int F(\omega) \frac{d^{2} W_{h}}{d \Omega d \omega} d \Omega d \omega .
$$

To solve this for the distribution in (22), we assume the e-beam is long enough that the spectral bandwidth is dominated by the e-beam rather than the undulator, $\omega_{b} \tau \gg 2 \pi N_{u}$. That is, the number of wavelengths in the e-beam is much greater than the number of undulator

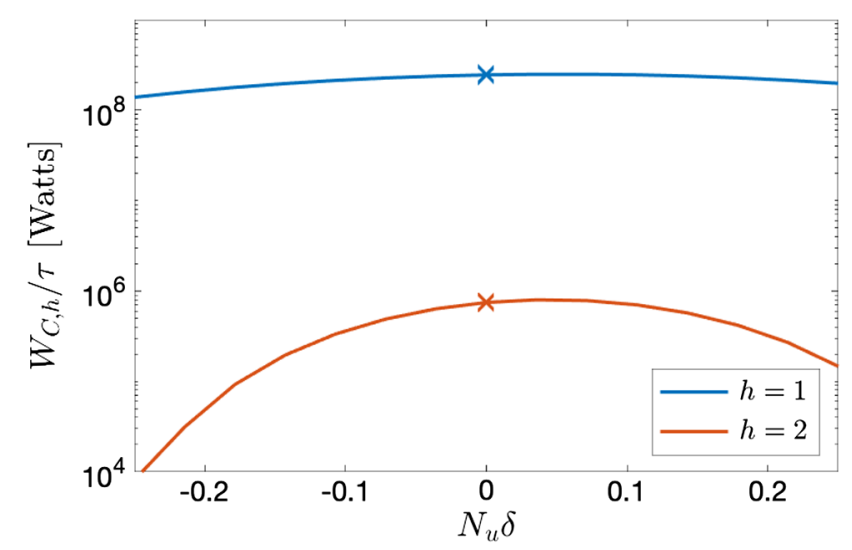

FIG. 5. Coherent emission peak power at different values of undulator detuning, $K \rightarrow K_{h}(1-\delta)$. Same parameters as Fig. 4 with $b_{h}=20 \%$ and $I_{0}=1 \mathrm{kA}$, but with $\sigma_{x}=25 \mu \mathrm{m}(N=1)$ for LCLS-II e-beams. Each $\mathbf{x}$ marks the coherent power emitted at zero detuning, calculated from Eq. (31). periods. In this case the bunching frequency just picks out a specific narrow portion of the undulator bandwidth, and the frequency integral can be solved easily with the approximation

$$
\operatorname{sinc}^{2}\left[\frac{\tau}{2}\left(\omega-\omega_{b}\right)\right] \rightarrow \frac{2 \pi}{\tau} \delta\left(\omega-\omega_{b}\right)
$$

If the bunching is at the same frequency as the on-axis undulator resonance $\omega_{b}=\omega_{h}(0)$ we obtain

$W_{C, h}=\tau \frac{2 \pi^{2} I_{0}^{2} b^{2} N_{u}^{2}}{c}\left(\frac{2 h K \gamma}{1+K^{2}}\right)^{2}$
$\int_{0}^{\pi} e^{-\left(\sigma_{x} \omega_{b} \theta / c\right)^{2}} \operatorname{sinc}\left[\pi N_{u} h \gamma_{z}^{2} \theta^{2}\right]^{2} J_{h-1}^{2}\left(\frac{2 h K \gamma \theta}{1+K^{2}}\right) \theta d \theta$

where $I_{0}=q N_{e} / \tau$ is the peak e-beam current.

Full solutions to this integral are cumbersome, but useful results can be found by series expansion of $J_{h-1}$ to lowest order. After some straightforward manipulation (outlined in Appendix B), the total energy emitted can then be written in a convenient form,

$$
W_{C, h}=\tau \frac{2 \pi^{2} I_{0}^{2} b_{h}^{2} N_{u}}{c}\left(\frac{K^{2}}{1+K^{2}}\right) \mathcal{F}_{h}(N) .
$$

Here $N=\omega \sigma_{x}^{2} / c L_{u}$ is the Fresnel number, $L_{u}=N_{u} \lambda_{u}$ is the undulator length, and $b_{h}$ is the bunching factor at each harmonic resonance. For the first few harmonics, the functions $\mathcal{F}_{h}(N)$ are

$$
\begin{aligned}
& \mathcal{F}_{1}(N)=\frac{2}{\pi}\left[\tan ^{-1}\left(\frac{1}{2 N}\right)+N \ln \left(\frac{4 N^{2}}{4 N^{2}+1}\right)\right] \\
& \mathcal{F}_{2}(N)=\frac{2}{\pi^{2} N_{u}} \frac{K^{2}}{1+K^{2}} \ln \left(\frac{4 N^{2}+1}{4 N^{2}}\right) \\
& \mathcal{F}_{3}(N)=\frac{27}{16}\left(\frac{1}{\pi N_{u}} \frac{K^{2}}{1+K^{2}}\right)^{2} \frac{1}{\pi N} \frac{1}{1+4 N^{2}} .
\end{aligned}
$$

These are plotted in Fig. 6. The factor $\mathcal{F}_{1}(N)$ for the fundamental frequency was obtained by Saldin et al., [38]. It has the simple limits $\mathcal{F}_{1}(N) \rightarrow 1 / 2 \pi N$ for $N \rightarrow \infty$ and $\mathcal{F}_{1}(N) \rightarrow 1$ for $N \rightarrow 0$. The factors $\mathcal{F}_{2}(N)$ and $\mathcal{F}_{3}(N)$ for the second and third harmonics incorporate the effect of the off-axis emission and show that the OAM emission energy for higher harmonics scales like $\left(1 / N_{u}\right)^{|l|}$. For $N \gg 1$, $\mathcal{F}_{2}(N)$ scales like $1 / N^{2}$, while $\mathcal{F}_{3}(N)$ scales like $1 / N^{3}$. Both diverge for $N \rightarrow 0$, so higher order corrections are required for an accurate description at $N<0.01$. Nevertheless, they yield a reasonable approximation for the OAM emission energy in many practical scenarios. It is evident that emission at harmonics is suppressed for large $N$, so transversely small e-beams produce coherent pulses with more photons. 


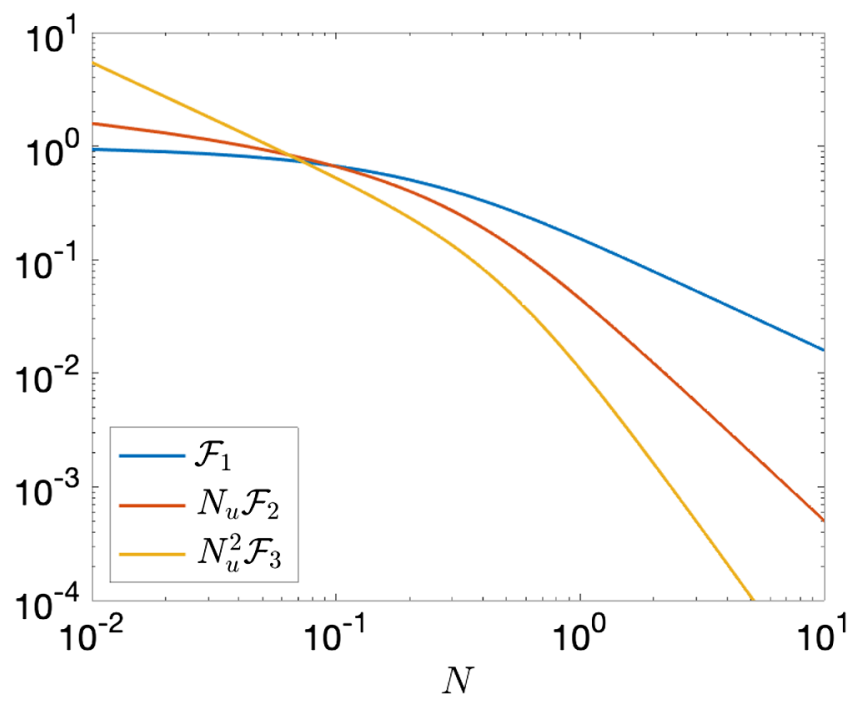

FIG. 6. Functions $\mathcal{F}_{h}(N)$ in Eq. (32). $K \gg 1$ is assumed.

\section{OAM AFTERBURNER}

Given Eqs. (31) and (32), we can now consider the coherent radiation energy at the fundamental frequency compared with the second harmonic frequency in an OAM afterburner configuration (see Fig. 7). Broadly speaking, an afterburner is an undulator positioned downstream of a high gain FEL [39]. For our purposes, the afterburner is a helical undulator, and the arrangement is similar to the proof-ofprinciple setup first described in Ref. [24]. The upstream FEL is used to drive bunching in the electron beam, either by lasing or by the reverse taper effect [40]. The bunched beam then radiates coherently in the afterburner. Previous versions of this arrangement have been used to produce coherent circularly polarized x-rays at the $h=1$ afterburner resonance [41], the energy output of which can be calculated from Eq. (31) with $\mathcal{F}_{1}(N)$. Here, in an OAM afterburner arrangement, the only change is the undulator $K$ of the afterburner. It is increased from $K_{1}$ to $K_{2}$ so that the

(a)

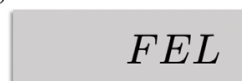

(b)

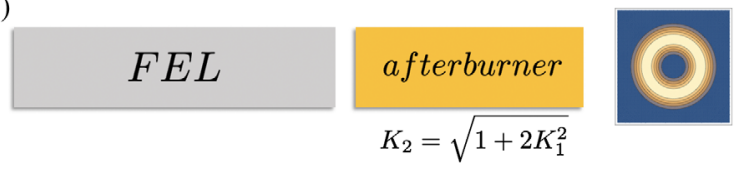

FIG. 7. OAM FEL afterburner scheme. In contrast to the typical case of coherent emission at the first harmonic (a), OAM light can be produced by tuning the helical undulator so that the incoming beam is bunched at the second harmonic (b). The upstream FEL uses reverse tapering to generate strong bunching with little radiation. Steering the electron beam prior to entry into the afterburner can transversely separate the OAM light from the FEL emission, as in Ref. [41].

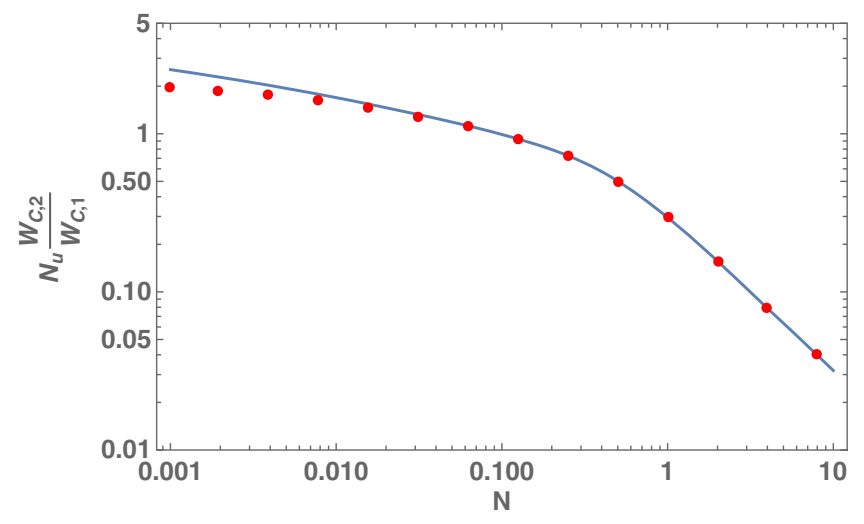

FIG. 8. Top: Ratio of radiated OAM energy at second harmonic to the fundamental in afterburner setup from Eq. (34). Beams with smaller $N=\omega \sigma_{x}^{2} / c L_{u}$ are favorable to produce more OAM energy. Here $K_{1}>1$ is assumed. Dots are from numerical integration of Eq. (28) using the full expression for the field in Eq. (5).

coherent emission is at the second harmonic of the undulator (i.e., from a Gaussian-like mode at $K_{1}$ to an OAM mode at $K_{2}$ ) but at the same frequency in both cases, $\omega_{1}=\omega_{2}$. Because the emission frequency is the same, the Fresnel number $N$ is the same. From Eq. (11) the relationship between the two values of $K$ is

$$
K_{2}^{2}=1+2 K_{1}^{2} \text {. }
$$

The interaction at the second harmonic is weaker than at the fundamental, so the energy radiated into the OAM mode is smaller. The ratio of the energy contained in the OAM at the second harmonic compared to the energy at the first harmonic is

$$
\frac{W_{C, 2}}{W_{C, 1}}=\frac{g\left(K_{1}\right)}{\pi N_{u}} \frac{\ln \left(\frac{4 N^{2}+1}{4 N^{2}}\right)}{\left[\tan ^{-1}\left(\frac{1}{2 N}\right)+N \ln \left(\frac{4 N^{2}}{4 N^{2}+1}\right)\right]}
$$

The ratio is shown in Fig. 8, and goes like $1 / \pi N_{u} N$ for $N>1$. For notational brevity we introduce the factor $g\left(K_{1}\right)=\left(1+2 K_{1}^{2}\right)^{2} / 4 K_{1}^{2}\left(1+K_{1}^{2}\right)$ which goes quickly to $g\left(K_{1}\right) \rightarrow 1$ for $K_{1}>1$, but goes like $g\left(K_{1}\right) \rightarrow 1 / 4 K_{1}^{2}$ for $K_{1} \ll 1$. As a useful point of reference, we see that for $K_{1}>1$, a Fresnel number of $N=0.1$ will produce OAM emission with total energy $1 / N_{u}$ that of the fundamental.

\section{CONCLUSIONS}

In the limit of small forward angles, many periods, and sufficiently strong undulator fields, the SAM and OAM components of radiation emission from a helical undulator are separable, additive, and aligned with the $z$-projection of OAM of the electrons on their helical orbit. For round electron beams, the coherent emission can have the same form as an LG mode, such that the total angular momentum of the coherent mode is $h \hbar$ per photon. The coherent 
emission energy is derived in simple form, and scales roughly like $1 / N_{u}^{h-1}$ for the harmonics, though beams with smaller Fresnel numbers can perform better. Results indicate that coherent, circularly polarized $\mathrm{x}$-rays at $1 \mathrm{~nm}$ wavelengths that carry $l= \pm \hbar$ of OAM can be produced at LCLS-II with 1 MW of pulse power with the baseline design. This can be increased to $20 \mathrm{MW}$ or more with smaller e-beams. If the emitted OAM mode can then be focused to a spot size of $w_{0}=0.1 \mu \mathrm{m}$, the peak electric field strengths reach 1 a.u., which potentially enables novel light-matter interaction experiments at soft x-rays.

\section{ACKNOWLEDGMENTS}

We thank J. Morgan, B. McNeil, and J. Cryan for helpful conversations. This work was supported by U.S. Department of Energy Contract No. DE-AC02-76SF00515 and Award No. 2017-SLAC-100382.

\section{APPENDIX A: FIELD EXPANSION}

The exponential in Eq. (5) can be written as a sum over Bessel functions,

$$
e^{i \omega(t-\hat{n} \cdot \vec{w} / c)}=e^{i \omega t\left(1-\beta_{z} \cos \theta\right)} \sum_{n, m}(-i)^{n}(\mp 1)^{m} J_{n}(\xi \sin \phi) J_{m}(\xi \cos \phi) e^{i(n+m) \omega_{u} t} .
$$

The total electric field then has several terms that make use of the following integral,

$$
\begin{aligned}
\int_{-\frac{N_{u} \pi}{\omega_{u}}}^{\frac{N_{u} \pi}{\omega_{u}}} e^{i \omega(t-\hat{n} \cdot \vec{w} / c)+i p \omega_{u} t} d t & =\frac{2 N_{u} \pi}{\omega_{u}} \sum_{n, m}(-i)^{n}(\mp 1)^{m} J_{n}(\xi \sin \phi) J_{m}(\xi \cos \phi) \operatorname{sinc}\left[N_{u} \pi\left(\frac{h \omega}{\omega_{h}(\theta)}+n+m+p\right)\right] \\
& \approx \frac{2 N_{u} \pi}{\omega_{u}}( \pm 1)^{h+p} e^{\mp i(h+p) \phi} J_{h+p}(\xi) \operatorname{sinc}\left[N_{u} \pi h\left(\frac{\omega}{\omega_{h}(\theta)}-1\right)\right]
\end{aligned}
$$

In the second step it has been assumed that $h=-(n+$ $m+p)$ and the summation theorem for Bessel functions has then been applied to collapse the sum. One should be aware that this approximation is common, but that it also removes some of the more subtle couplings between angles and frequencies. As a result, it describes radiation profiles that can be artificially more azimuthally symmetric (independent of $\phi$ ) than when compared to the exact solution, especially for higher harmonics. In most cases of interest for lower harmonics, however, the deviation is small and this is a good approximation.

\section{APPENDIX B: ENERGY INTEGRAL}

Solutions to the total energy integral in Eq. (30) are obtained with the help of two relations. The first is the integral representation of the Bessel function,

$$
J_{n}(x)=\frac{1}{2 \pi} \int_{-\pi}^{\pi} e^{-i n \tau+i x \sin \tau} d \tau .
$$

The second is the Fourier transform of the $\operatorname{sinc}^{2}(x)$ function

$$
\operatorname{sinc}^{2}(\omega T / 2)=\int_{-1}^{1}(1-|\xi|) e^{i \omega T \xi} d \xi
$$

where the triangle function is defined as $\operatorname{tri}(\xi)=1-|\xi|$ for $|\xi|<1$, and $\operatorname{tri}(\xi)=0$ otherwise. After some straightforward manipulations, the integral in Eq. (30) can then be written as

$$
\begin{gathered}
\int_{0}^{\infty} e^{-(\nu \theta)^{2}} \operatorname{sinc}\left[\eta \theta^{2}\right]^{2} J_{h-1}^{2}(\alpha \theta) \theta d \theta \\
=\frac{\pi}{4 \eta} \sum_{n=0} \frac{\mathcal{I}_{h, n}}{(2 n) !}\left(\frac{\alpha^{2}}{4 \eta}\right)^{n} \frac{\partial^{n}}{\partial N^{n}} \mathcal{F}_{1}(N)
\end{gathered}
$$

where $\nu=\sigma_{x} \omega_{h} / c, \eta=\pi N_{u} h \gamma_{z}^{2}, \alpha=2 h \gamma_{z}^{2} K / \gamma, \nu^{2} / 2 \eta=$ $2 N$. The sum originates from a series expansion over small values of the Bessel argument. For the fundamental harmonic, the lowest order term is the exact integral

$\int_{0}^{\infty} e^{-\left(\sigma_{x} \omega_{b} \theta / c\right)^{2}} \operatorname{sinc}\left[\pi N_{u} \theta^{2} \gamma_{z}^{2}\right]^{2} \theta d \theta=\frac{1}{4 N_{u} \gamma_{z}^{2}} \mathcal{F}_{1}(N)$

where

$$
\mathcal{F}_{1}(N)=\frac{2}{\pi}\left[\tan ^{-1}\left(\frac{1}{2 N}\right)+N \ln \left(\frac{4 N^{2}}{4 N^{2}+1}\right)\right]
$$

as also obtained in Ref. [38]. Values for individual harmonics and different terms in the sum are calculated by the integral

$$
\mathcal{I}_{h, n}=\frac{1}{(2 \pi)^{2}} \int_{-\pi}^{\pi} d \tau d \tau^{\prime} e^{-i(h-1)\left(\tau-\tau^{\prime}\right)}\left(\sin \tau-\sin \tau^{\prime}\right)^{2 n} .
$$

The first few terms for the fundamental are $\mathcal{I}_{1,0}=1$, $\mathcal{I}_{1,1}=1$, and $\mathcal{I}_{1,2}=9 / 4$. For the second harmonic, $\mathcal{I}_{2,0}=0, \mathcal{I}_{2,1}=-1 / 2$, and $\mathcal{I}_{2,2}=-3 / 2$, and for the third 
harmonic, $\mathcal{I}_{3,0}, \mathcal{I}_{3,1}=0$, and $\mathcal{I}_{3,2}=3 / 8$, and so on. The lowest order contributor for the harmonic $h$ corresponds to the $h-1$ derivative of $\mathcal{F}_{1}(N)$.

In the practical case of small coefficients

$$
\frac{\alpha^{2}}{4 \eta}=\frac{h}{\pi N_{u}} \frac{K^{2}}{1+K^{2}} \ll 1
$$

the higher order terms become ever smaller, so for each harmonic we can take only the first nonzero term in the sum. Calculation is aided with the help of the relations

$$
\frac{\partial}{\partial N} \mathcal{F}_{1}(N)=\frac{2}{\pi} \ln \left(\frac{4 N^{2}}{1+4 N^{2}}\right),
$$

and

$$
\frac{\partial^{2}}{\partial N^{2}} \mathcal{F}_{1}(N)=\frac{4}{\pi N} \frac{1}{1+4 N^{2}}
$$

[1] W. Heitler, On the radiation emitted by a multipole and its angular momentum, Math. Proc. Cambridge Philos. Soc. 32, 112 (1936).

[2] L. Allen, M. W. Beijersbergen, R. J. C. Spreeuw, and J. P. Woerdman, Orbital angular momentum of light and the transformation of Laguerre-Gaussian laser modes, Phys. Rev. A 45, 8185 (1992).

[3] S. M. Barnett, L. Allen, R. P. Cameron, C. R. Gilson, M. J. Padgett, F. C. Speirits, and A. M. Yao, On the natures of the spin and orbital parts of optical angular momentum, J. Opt. 18, 064004 (2016).

[4] L. Allen, S. M. Barnett, and M. J. Padgett, Optical Angular Momentum (Institute of Physics Pub., Bristol, Philadelphia, 2003).

[5] A. M. Yao and M. J. Padgett, Orbital angular momentum: origins, behavior and applications, Adv. Opt. Photonics 3, 161 (2011).

[6] S. Franke-Arnold, L. Allen, and M. Padgett, Advances in optical angular momentum, Laser Photonics Rev. 2, 299 (2008).

[7] G. Molina-Terriza, J. P. Torres, and L. Torner, Twisted photons, Nat. Phys. 3, 305 (2007).

[8] M. Babiker, D. L. Andrews, and V. E. Lembessis, Atoms in complex twisted light, J. Opt. 21, 013001 (2018).

[9] A. Alexandrescu, D. Cojoc, and E. D. Fabrizio, Mechanism of Angular Momentum Exchange between Molecules and Laguerre-Gaussian Beams, Phys. Rev. Lett. 96, 243001 (2006).

[10] M. van Veenendaal and I. McNulty, Prediction of Strong Dichroism Induced by X Rays Carrying Orbital Momentum, Phys. Rev. Lett. 98, 157401 (2007).

[11] M. Babiker, C. R. Bennett, D. L. Andrews, and L. C. D. Romero, Orbital Angular Momentum Exchange in the
Interaction of Twisted Light with Molecules, Phys. Rev. Lett. 89, 143601 (2002).

[12] W. Löffler, D. J. Broer, and J. P. Woerdman, Circular dichroism of cholesteric polymers and the orbital angular momentum of light, Phys. Rev. A 83, 065801 (2011).

[13] R. Mathevet, B. V. de Lesegno, L. Pruvost, and G. L. J. A. Rikken, Negative experimental evidence for magnetoorbital dichroism, Opt. Express 21, 3941 (2013).

[14] C. T. Schmiegelow, J. Schulz, H. Kaufmann, T. Ruster, U. G. Poschinger, and F. Schmidt-Kaler, Transfer of optical orbital angular momentum to a bound electron, Nat. Commun. 7, 12998 (2016).

[15] F. Giammanco, A. Perona, P. Marsili, F. Conti, F. Fidecaro, S. Gozzini, and A. Lucchesini, Influence of the photon orbital angular momentum on electric dipole transitions: negative experimental evidence, Opt. Lett. 42, 219 (2017).

[16] P. Emma et al., First lasing and operation of an ångstromwavelength free-electron laser, Nat. Photonics 4, 641 (2010).

[17] T. Ishikawa et al., A compact X-ray free-electron laser emitting in the sub-ångström region, Nat. Photonics 6, 540 (2012).

[18] H.-S. Kang et al., Hard X-ray free-electron laser with femtosecond-scale timing jitter, Nat. Photonics 11, 708 (2017).

[19] S. Sasaki and I. McNulty, Proposal for Generating Brilliant X-Ray Beams Carrying Orbital Angular Momentum, Phys. Rev. Lett. 100, 124801 (2008).

[20] W. Colson, The nonlinear wave equation for higher harmonics in free-electron lasers, IEEE J. Quantum Electron. 17, 1417 (1981).

[21] E. Allaria, F. Curbis, M. Coreno, M. Danailov, B. Diviacco, C. Spezzani, M. Trovó, and G. DeNinno, Experimental Characterization of Nonlinear Harmonic Generation in Planar and Helical Undulators, Phys. Rev. Lett. 100, 174801 (2008).

[22] G. Geloni, E. Saldin, E. Schneidmiller, and M. Yurkov, Theory of nonlinear harmonic generation in free-electron lasers with helical wigglers, Nucl. Instrum. Methods Phys. Res., Sect. A 581, 856 (2007).

[23] J. Bahrdt, K. Holldack, P. Kuske, R. Müller, M. Scheer, and P. Schmid, First Observation of Photons Carrying Orbital Angular Momentum in Undulator Radiation, Phys. Rev. Lett. 111, 034801 (2013).

[24] E. Hemsing, M. Dunning, C. Hast, T. Raubenheimer, and D. Xiang, First Characterization of Coherent Optical Vortices from Harmonic Undulator Radiation, Phys. Rev. Lett. 113, 134803 (2014).

[25] P. R. Ribič, B. Rösner, D. Gauthier, E. Allaria, F. Döring, L. Foglia, L. Giannessi, N. Mahne, M. Manfredda, C. Masciovecchio, R. Mincigrucci, N. Mirian, E. Principi, E. Roussel, A. Simoncig, S. Spampinati, C. David, and G. De Ninno, Extreme-Ultraviolet Vortices from a Free-Electron Laser, Phys. Rev. X 7, 031036 (2017).

[26] M. Katoh, M. Fujimoto, N. S. Mirian, T. Konomi, Y. Taira, T. Kaneyasu, M. Hosaka, N. Yamamoto, A. Mochihashi, Y. Takashima, K. Kuroda, A. Miyamoto, K. Miyamoto, and S. Sasaki, Helical Phase Structure of Radiation from an Electron in Circular Motion, Sci. Rep. 7, 6130 (2017). 
[27] A. Afanasev and A. Mikhailichenko, On generation of photons carrying orbital angular momentum in the helical undulator, arXiv:1109.1603.

[28] M. Katoh, M. Fujimoto, H. Kawaguchi, K. Tsuchiya, K. Ohmi, T. Kaneyasu, Y. Taira, M. Hosaka, A. Mochihashi, and Y. Takashima, Angular Momentum of Twisted Radiation from an Electron in Spiral Motion, Phys. Rev. Lett. 118, 094801 (2017).

[29] T. Kaneyasu, Y. Hikosaka, M. Fujimoto, T. Konomi, M. Katoh, H. Iwayama, and E. Shigemasa, Limitations in photoionization of helium by an extreme ultraviolet optical vortex, Phys. Rev. A 95, 023413 (2017).

[30] A. Picón, J. Mompart, J. R. V. de Aldana, L. Plaja, G. F. Calvo, and L. Roso, Photoionization with orbital angular momentum beams, Opt. Express 18, 3660 (2010).

[31] J. D. Jackson, Classical Electrodynamics, 3rd ed. (J. Wiley and Sons, New York, 1999).

[32] S. M. Barnett and L. Allen, Orbital angular momentum and nonparaxial light beams, Opt. Commun. 110, 670 (1994).

[33] Interestingly, at a fixed point in time, the electromagnetic field vector evolves along $z$ in the opposite sense as the electrons in the undulator. See Ref. [34] page 230.

[34] E. Hemsing, Generation and amplification of coherent radiation with optical orbital angular momentum in a freeelectron laser, Ph.D. thesis, University of California Los Angeles, 2011.

[35] E. Hemsing, A. Knyazik, M. Dunning, D. Xiang, A. Marinelli, C. Hast, and J. B. Rosenzweig, Coherent optical vortices from relativistic electron beams, Nat. Phys. 9, 549 (2013).

[36] R. W. Schoenlein, New science opportunities enabled by LCLS-II x-ray lasers, SLAC National Accelerator Laboratory Technical Report No. SLAC-R-1053, 2015.

[37] For a transversely elliptical e-beam, it is straightforward to show that the form factor is $F(\omega) \propto$ $\exp \left[-\frac{\omega^{2}}{c^{2}} \sin ^{2} \theta\left(\sigma_{x}^{2} \cos ^{2} \phi+\sigma_{y}^{2} \sin ^{2} \phi\right)\right]$. In contrast with Eq. (26), it has a $\phi$ dependence that can impact the symmetry and purity of the coherently emitted OAM modes. This affects the subsequent analysis and is a topic of future study, but suggests that the e-beam should, in general, be made as round as possible in the afterburner.

[38] E. L. Saldin, E. A. Schneidmiller, and M. V. Yurkov, Statistical and coherence properties of radiation from $\mathrm{x}$-ray free-electron lasers, New J. Phys. 12, 035010 (2009).

[39] E. L. Saldin, E. A. Schneidmiller, and M. V. Yurkov, Optical afterburner for an X-ray free electron laser as a tool for pump-probe experiments, Phys. Rev. Accel. Beams 13, 030701 (2010).

[40] E. A. Schneidmiller and M. V. Yurkov, Obtaining high degree of circular polarization at X-ray free electron lasers via a reverse undulator taper, Phys. Rev. Accel. Beams 16, 110702 (2013).

[41] A. A. Lutman et al., Polarization control in an X-ray freeelectron laser, Nat. Photonics 10, 468 (2016). 